\title{
Die Überwindung verstandesmäßiger Freiheitskonzeptionen in Hegels „Herrschaft und Knechtschaft“
}

\author{
SEBASTIAN OSTRITSCH \\ Universität Stuttgart
}

\section{EINLEITUNG}

Seit den Aufsehen erregenden Experimenten Benjamin Libets zur Willensfreiheit werden unsere alltäglichen Auffassungen von Freiheit, Handlung, Selbstbestimmtheit und Verantwortlichkeit von Neurobiologen und naturalistischen Philosophen in Frage gestellt. Gemeinhin gilt der Kompatibilismus innerhalb der gegenwärtigen philosophischen Freiheitsdebatte als aussichtsreichster Kandidat für eine Versöhnung der lebensweltlichen und der szientistischen Auffassung vom Menschen. Während der Determinismus für eine Streichung des Begriffs menschlicher Freiheit optiert und sich somit auf die kontraintuitive Position festlegt, menschliche Freiheit sei reine Illusion, verpflichtet sich der Libertarianismus, der zwar die Intuition des Common Sense bezüglich Freiheit und Verantwortlichkeit philosophisch stützen kann, auf eine Theorie, die dem für die Naturwissenschaften wesentlichen heuristischen Prinzip einer durchgängigen Kausaldetermination widerspricht. In beiden Fällen werden fest verankerte Überzeugungen in Frage gestellt. Determinismus und Libertarianismus stellen uns vor die Entscheidung, uns entweder von unserer Auffassung von wissenschaftlicher Praxis oder von unserem alltäglichen Selbstverständnis zu verabschieden. Der Kompatibilismus hingegen legt nahe, diese Entscheidung überhaupt nicht treffen zu müssen.

Für eine hegelianische Alternative zu der Trias von Determinismus, Libertarianismus und Kompatibilismus wird im Folgenden in drei Schritten argumentiert:

1. Das sich aus der gleichzeitigen Annahme der Gültigkeit von Determinismus und Freiheit ergebende Problem soll anhand des von Peter Bieri formulierten Trilemmas in seiner Grundstruktur aufgezeigt werden.

2. Anhand der Lektüre des Kapitels „Kraft und Verstand“ aus der Phänomenologie des Geistes, und unter besonderer Berücksichtigung des 
„Spiels der Kräfte“, soll die Beliebigkeit objektstufiger Modellierungen des Verhältnisses von menschlicher Freiheit und Determinismus erwiesen werden.

3. Ausgehend von einer intrasubjektiven Deutung des „Herrschaft-Knechtschaft" Kapitels soll abschließend ein hegelianischer Ausweg aus der argumentativen Pattsituation zwischen den gängigen Positionen zum Freiheitsproblem dargestellt werden.

\section{Die Grundstruktur des Freiheitsproblems}

Peter Bieri hat das basale Trilemma der Philosophy of Mind wie folgt formuliert:

a. Mentale Phänomene sind nicht-physische Phänomene.

b. Mentale Phänomene sind im Bereich physischer Phänomene kausal wirksam.

c. Der Bereich physischer Phänomene ist kausal geschlossen (Vgl. Bieri 1997: 5).

Für die Freiheitsdebatte lässt sich das Bieri-Trilemma wie folgt neu formulieren:

d. Freie Handlungen sind nicht-naturgesetzlich determinierte Phänomene.

e. Freie Handlungen bewirken physikalische Zustände und Ereignisse in der Welt.

f. Der Bereich der naturgesetzlich determinierten Phänomene ist geschlossen.

Die gängigen Positionen zu diesem Problem haben gemein, dass sie eine der lebensweltlich oder wissenschaftspraktisch evidenten Prämissen aufgeben. Sie unterscheiden sich lediglich darin, welche der Prämissen sie für den Erhalt der anderen opfern:

i. Der Determinismus bestreitet die lebensweltlich fest verankerte These, dass freie Handlungen im Bereich naturgesetzlich determinierter Phänomene wirksam sind. Da es streng genommen keine freien Handlungen gibt, sondern lediglich scheinbar freie Handlungen, die sich bei genauerer Beobachtung als naturgesetzlich determinierte körperliche Abläufe entpuppen, wird der Determinist auch die erste Prämisse aufgeben, indem er sie für sinnlos erklärt.

ii. Der Libertarianismus vertritt die Gegenthese, indem er behauptet, der Bereich naturgesetzlich determinierter Phänomene sei nicht geschlossen. Dadurch opfert der Libertarier das zentrale forschungsleitende Prinzip moderner naturwissenschaftlicher Forschung für den Erhalt der Lebenswelt.

iii. Der Kompatibilismus mag angesichts des hohen Preises, den Determi- 
nismus und Libertarianismus zu zahlen haben, als die einzig befriedigende Position innerhalb dieser Diskussion erscheinen: Aus epistemischer Perspektive der handelnden Personen ließe sich sowohl Determinismus als auch Freiheit denken.Zwar sei die Welt an sich und somit auch das handelende Subjekt als ein Teil von ihr vollständig determiniert, für den Akteur sei diese kausale Vollbestimmtheit jedoch irrelevant, da nicht zugänglich, was dazu führe, dass er immer noch gemäß einer als-ob Freiheit handeln könne.

\section{III. „KRAFt und Verstand“ und die Beliebigkeit der Modellierung VON FREIHEIT UND DETERMINISMUS}

Im Folgenden soll gezeigt werden, wie Hegels Kapitel „Kraft und Verstand“ in der Phänomenologie des Geistes als Kritik der drei skizzierten Positionen gelesen werden kann. Dazu bedarf es zunächst einer weiteren Umformulierung des Bieri-Trilemmas, die zeigt, dass die Determinismus-Freiheit Debatte als ein Streit um die Stellung des Ich aufzufassen ist. Aus lebensweltlicher Perspektive wird das Ich als ein nicht fremdbestimmtes und somit freies Subjekt gedacht. Im Bereich naturgesetzlicher Determination wird das Ich als ein „von außen“" bestimmtes Objekt betrachtet. In seiner Rolle als Objekt gilt das Ich als auf neurophysiologische Strukturen und Prozesse reduzierbar.

g. Das Ich ist kein fremdbestimmtes Objekt

h. Das Ich bestimmt sich selbst

i. Alles ist Objekt naturgesetzlicher Bestimmung

Die zentrale These des Verstandeskapitels ist, dass kausale Modellierungen, wie sie in der Determinismus-Freiheit Debatte vorzufinden sind, beliebig sind. Hegel drückt diese Beliebigkeit mit der Metapher des „Spiels der Kräfte“ aus. In „Kraft und Verstand“ fragt Hegel im Anschluss an das Kapitel „Die Wahrnehmung“ nach denjenigen (begrifflichen) Regeln, unter denen Wahrnehmungen stattfinden und schließt somit an die kantische Theorie des Verstandes als dem Vermögen der Regeln an. Zentraler Begriff für die Regulierung von Wahrnehmung ist „Kraft“ (PhG: 95). Kraft als das Prinzip der Bewegung soll ermöglichen, die in der Wahrnehmung selbst unerklärlichen Veränderungen von Eigenschaften eines gleichbleibenden Dinges verständlich zu machen. Allerdings lässt sich Kraft nur über die wechselseitig präsupponierenden Oppositionsinstanzen einer wirkenden (sollizitierenden) Kraft und einer potentiellen (sollizitierten) Kraft begreifen. Hegel greift also zu Beginn des Verstandeskapitels die Aristotelische Lehre von dynamis und energeia mit seinen Termini der sollizitierten und sollizitierenden Kraft auf. Die wesentliche Einsicht des Kapitels besteht darin, dass aus prinzipiellen Gründen nicht zu erkennen ist, wie die dynamis-energeia Relation zu denken ist, d.h. welches der beiden Relata als dynamis und welches als energeia aufgefasst werden muss. Der 
Grund dafür ist simpel: Sollizitierende und sollizitierte Kraft sind austauschbare Bestimmungen; was als wirkende und was als potentielle Kraft gilt, erscheint willkürlich, da eine wirkenden Kraft nur sollizitierend ist, „dadurch, daß sie sollizitiert wird" (PhG: 98). Anstatt den Wechsel der Eigenschaften an einem Ding erklären zu können, unterliegt Kraft selbst der Veränderung. Sie selbst ist ja nur als Wechsel sollizitierender und sollizitierter Kraft:

„Das sollizitierende ist, zum Beispiel, als allgemeines Medium, und dagegen das Sollizitierte als zurückgedrängte Kraft gesetzt; aber jenes ist allgemeines Medium selbst nur dadurch, daß das andere zurückgedrängte Kraft ist; oder diese ist vielmehr das Sollizitierende für jenes, und macht dasselbe erst zum Medium“ (ibid.).

Anstatt Veränderung erklären zu können, stellt sich vielmehr erneut die Frage nach den unveränderlichen Regeln dieses Spiels der Kräfte.

Für die Determinismus-Freiheit Debatte heißt dies: (1) Ob das Ich Subjekt, d.h. die wirkende Kraft (energeia), oder Objekt, d.h. Kraft auf die eingewirkt wird (dynamis) ist, lässt sich nicht erkennen, sondern nur dogmatisch festlegen. (2) Da sich der Kompatibilismus auf der selben argumentativen Ebene bewegt wie Determinismus und Libertarianismus, kann sein Versöhnungversuch lediglich als schlechter Kompromiss des Verstandes begriffen werden, der ständig dem Oszillieren zwischen den beiden Extrempositionen unterworfen ist. Er verschiebt die Frage nach der Konzeptualisierung des Verhältnisses von Ich als Subjekt und von Ich als Objekt lediglich um eine Ebene. Zu fragen bleibt nämlich, von welchem Standpunkt aus die Möglichkeit von Freiheit konstatiert wird. Wie die für den Kompatibilismus typische Rede von einer ,als-ob Freiheit“ erhellt, ist dies für gewöhnlich der Standpunkt des Determinismus. Damit aber ergibt sich erneut die Frage nach der Legitimation dieses Standpunktes.

\section{Anerkennen statt Erkennen: Die intrasubjektive Lesart des He- RRSCHAFT UND KNECHTSCHAFT KAPITELS}

Das Scheitern des Erkennens im Verstandeskapitel bedeutet aus der Sicht der Phänomenologie nicht das Ende der kategorialen Bildungsreise des Geistes. Vielmehr ergibt sich zu Beginn des Selbstbewusstseinskapitels ein neuer Modus der Welterschließung: das Anerkennen. Der Schlüssel dafür, das Selbstbewusstseinskapitel als Lösungsvorschlag zur Determinismus-Freiheit Debatte aufzufassen, besteht in einer intrasubjektiven Lesart. Diese fasst den Herrn und den Knecht als zwei subjektimmanente Momente auf. Für die Ablehnung der gängigen intersubjektiven Interpretation, die das Herr-Knecht Verhältnis auf zwei Subjekte bezieht, sprechen wesentlich zwei Gründe:

1. Aus werkimmanenter Perspektive ist anzunehmen, dass Hegel nicht gegen das für die Phänomenologie konstitutive Prinzip verstößt, die zentralen Begriffe dialektisch auseinander zu entwickeln. Auf der Stufe 
des Selbstbewusstseinskapitels die Einführung mehrerer Subjekte, also die Kategorie der Intersubjektivität, erkennen zu wollen, widerspricht genau diesem Prinzip: Denn damit würde die Kategorie der Intersubjektivität in den Bereich des subjektiven Geistes vorgezogen.

2. Außerdem ist in argumentationslogischer Hinsicht anzunehmen, dass Hegel im Selbstbewusstseinskapitel keine petitio principii begeht. Genau dies müsste man ihm aber mit einer intersubjektiven Lesart unterstellen: Das Selbstbewusstsein eines Subjekts soll durch das Selbstbewusstsein eines anderen Subjekts ermöglicht werden, wodurch aber vorausgesetzt wird, was es zu begründen gilt.

Die intrasubjektive Deutung versteht die Rede von Herr und Knecht sowie die damit einhergehende idiosynkratische Rede von ,zwei Selbstbewusstsein" [sic!] (PhG: 129) als Allegorie der kantischen Unterscheidung zwischen transzendentalem und empirischem Ich. Ein solcher Deutungsansatz kann für sich beanspruchen, den Übergang vom Verstandeskapitel, das explizit mit der kantischen transzendentalen Apperzeption endet, ebenso verständlich zu machen, wie Hegels Kritik an der ,bewegungslose[n] Tautologie des: Ich bin Ich“ (PhG: 121) zu Beginn des Selbstbewusstseinskapitels (vgl. McDowell 2003).

Hier liegt nun die Interpretation der Metaphern von Herr und Knecht nahe, die unter anderem von Pirmin Stekeler-Weithofer vertreten wird (vgl. Stekeler-Weithofer 2008). Entsprechend der aristotelischen Konzeption der Seele als energeia, einer sich verwirklichenden inneren Kraft, die sich im Leib als ihrem äußeren manifestiert, ist die „Herr-Knecht-Allegorie [...] das uralte Bild vom Bewußtsein als Herr über den Leib“ (ibid.: 221). Das reine Selbstbewußtsein, die Seele, die bloße Instanz der Tätigkeit ist, nennt Hegel „Herr". „Seele“ entspricht hier dem weiten, alltäglichen, Begriff von Seele als derjenigen Instanz, die ,,in uns“ die Handlungsvorgaben macht und ist somit nicht mit dem engeren Seelenbegriff, den Hegel in der Enzyklopädie einführt, zu identifizieren (Vgl. Enz: § 388). Die Seele bezieht sich auf den Leib, indem sie Vorgaben, Absichten, Wünsche, „Begierden“(PhG: 135), kurz: begriffliche Normen für den Umgang mit der Welt vorgibt. Insofern ist es auch gerechtfertigt, die Knechtseite des Bewusstseins mit dem Leib zu identifizieren, da dieser eingespannt ist zwischen den Vorgaben der Seele und der Welt der Dinge, auf die er sich gemäß den Vorgaben tätig beziehen soll.

Hegel behauptet nun überraschenderweise, dass die ,Wahrheit des selbstständigen Bewußtseins [des Herrn][...] das knechtische Bewusstsein“" (PhG: 134) sei. Diesen Satz gilt es nun zu explizieren.

Hegel beginnt die Passagen zu Herrschaft und Knechtschaft mit der berühmten Formulierung:

„Das Selbstbewußtsein ist an und für sich, indem, und dadurch, daß es für ein 
anderes an und für sich ist; d.h. es ist nur als Anerkanntes“(PhG: 127).

Der epistemische Modus des Anerkennens stellt eine Alternative zum Erkennen dar, das sich im Zuge des Verstandeskapitels als unbrauchbar erwiesen hatte, das Verhältnis des Bewusstseins zu sich selbst zu modellieren. Die Relata der Anerkennensrelation sind aber nicht zwei Subjekte, sondern die beiden Bewusstseinsmomente des Herrn (Seele) und des Knechts (Leib). Ausgangspunkt für die dialektische Entwicklung ist der Umstand, dass der Leib die Seele und ihre Vorgaben anerkennen muss, da ohne die Seele die für den Leib konstitutive Einheit nicht gewährleistet werden kann: Ohne die Seele gäbe es lediglich ein nicht zu fassendes Gewirr von „vielfärbigen“ (KrV: B 134) empirischen Vorstellungen. Die Seele hingegen scheint frei von solchen Abhängigkeiten zu sein und gilt somit ihrem Begriff nach als „selbstständig“. Mitbedacht werden muss aber auch die Welt, auf die sich das Ich bezieht. Das bisher entwickelte Herr-Knecht-Verhältnis liest sich also wie folgt: Der Leib bezieht sich unter Anerkennung der Seele auf die Welt (das Ding), während die Seele sich mittels des Leibes auf die Welt bezieht. Dem Bezug des Leibes auf die Welt gibt Hegel den Titel „Arbeit“. Der Gedanke, dass innerhalb dieser Konstellation die Seele ohne den Leib auskommen könnte, kann sich nicht bewähren. Der Anspruch auf die Einheit des Selbstbewusstsein - ,die befriedigte Begierde“" des Herrn - lässt sich nur einlösen, wenn der Leib das zu synthetisierende Material dem Herrn darbietet. Die Seele ist ein bloßer „Papiertiger“ (Stekeler-Weithofer 2008: 228), wenn der Leib die Welt nicht bearbeitet. In diesem Sinne ist auch der Satz Hegels zu verstehen:

„In dieser Erfahrung wird es dem Selbstbewußtsein, daß ihm das Leben [i.e. die Mannigfaltigkeit der Weltinhalte, S.O.] ebenso wesentlich ist als das reine Selbstbewußtsein“ (PhG: 132).

Die Seele (der Herr) ist ihrem Anspruch nach selbstständiges, unabhängiges Selbstbewusstsein. Es hat sich aber gezeigt, dass sie diesen Anspruch nicht einlösen kann: Ihre Wahrheit ist der Leib (der Knecht).

Eine Theorie des Selbstbewusstseins lässt sich zwar nicht ausgehend vom Herrn entwickeln, dafür aber vom Knecht aus. Entscheidend hierfür ist die Arbeit des knechtischen Selbstbewusstseins, also sein tätiges Verhältnis zur Welt der Dinge. Hegel charakterisiert die Arbeit als , gehemmte Begierde, aufgehaltenes Verschwinden“(PhG: 135) und als „Bildung“ (ibid.). Gehemmt ist diese Begierde, weil der Leib die modale Differenz zwischen der Möglichkeit, dem bloßen Anspruch, so und nicht anders zu verfahren und der Wirklichkeit, d.h. seiner konkreten Realisierung, auf Grund der Widerständigkeit der Welt nicht zum Verschwinden bringen kann.

Dadurch bekommt der Leib als tätiges Selbstbewusstsein vorgeführt, was es heißt, ein Selbstbewußtsein zu sein: Er erfährt die Differenz zwischen 
der Formvorgabe des Herrn und dessen Verwirklichung und somit sich selbst als Urheber, d.h. als Subjekt des Verwirklichens. Hegel drückt dies so aus: „Diese negative Mitte oder das formierende Tun ist zugleich die Einzelheit oder das reine Fürsichsein des Bewußtseins, welches nun in der Arbeit außer es in das Element des Bleibens tritt; das arbeitende Bewußtsein kommt also hierdurch zur Anschauung des selbstständigen als seiner selbst" (PhG: 135). So wird aus dem zuerst nur „fremde[n] Sinn“ (PhG: 136), den der Leib bei der Formvorgabe der Seele erfährt, ein „eigener Sinn“ (PhG: 136). Das Selbstbewusstsein ist somit die über die Differenzerfahrung (von Formvorgabe und Umsetzung im Werk) des Leibes gewonnene Einheit von Ich als Subjekt (das Moment des Herrn, d.i. die Seele) und Ich als Objekt (das bearbeitete Ding, d.i. der Körper im Gegensatz zum Leib). Die Selbstbewusstseinsstruktur lässt sich somit als Instantiierung der dialektischen Figur der Identität von Identität und NichtIdentität begreifen.

\section{Beschluss}

Projizieren wir die Frage nach der „Stellung“ des Ich zurück auf das Verhältnis von Freiheit und Determinismus, können wir sehen, dass die beiden gegensätzlichen Bestimmungen von Freiheit und Determinismus von Hegel als Relata in einer sie umgreifenden Beziehung aufgelöst werden, die selbst die der Freiheit ist. Bleibt aber bei allem hegelianischen hin und her nicht trotzdem das Problem bestehen, dass das Subjekt einerseits als ein durch Naturkausalität durchgängig bestimmter Gegenstand erscheint (als Objekt) und anderseits als ein dieser Bestimmung widersprechender freier Zwecksetzer (als Subjekt) gedacht wird?

Das ist nicht der Fall, denn die in der Relation des Anerkennens aufgelösten Momente von

„Regularität [i.e. die Normvorgabe des Herrn, S.O.], Subjektivität [erschlossen über die Differenzerfahrung von Vorgabe und dessen Realisierung des Knechtes, S.O.] und Handlungsmaterie [verstanden als die die Selbsterkenntnis ermöglichende Widerständigkeit der Welt, S.O.]“ (Hubig 1985: 168f.)

ermöglichen erst den (eigentlich zu vermeidenden) verstandesmäßigen Gebrauch von Freiheit und Determinismus: Beide sind, wie ihre Verortung innerhalb des Anerkennungsverhältnisses zeigt, nicht derart aufzufassen, dass Gegenstände „bereitlägen“, die als freie oder naturkausal determinierte nur noch zu erkennen, d.h. lediglich unter die ihnen entsprechenden begrifflichen Regeln zu bringen wären. Vielmehr gilt die logische Priorität des Anerkennungsverhältnisses einer freien personalen Identität. Freiheit taucht somit im Anerkennen als eine Doppelte auf. Einmal als Moment des Verhältnisses, i.e. die Herr-Seite, und einmal als die über den Knecht erschlossene übergreifende 
Relation selbst.

Erst ex post, d.h. nach der Realisierung anerkannter Zwecksetzungen, weiß sich das Selbstbewusstsein als freies Selbstbewusstsein. Die Freiheit als Möglichkeit, sich selbst so oder anders zu bestimmen, ist für jede Zweckrealisierung immer schon vorausgesetzt, kann in ihrem Möglichkeitscharakter vom Subjekt aber erst rückwirkend, d.h. über die getane „Arbeit“, als die seine begriffen werden. Die kantische Frage, wie der Wille als ein Vermögen gedacht werden kann, das ,der Vorstellung gewisser Gesetze gemäß, sich selbst zum Handeln zu bestimmen“(GMS: AA 427) vermag, beantwortet Hegel also durch den Hinweis, dass eine Regel nur als anerkannte gültig ist.

Freiheit als ein intrasubjektives Anerkennungsverhältnis hebt sowohl das Determiniertwerden des Leibes als auch die freie Tätigkeit des Geistes in einer sie umgreifenden Einheit auf. Die Dichotomie „Ich als Subjekt“ oder „Ich als Objekt" wird durch die übergreifende Relation des Ich als Subjekt, die die Vorstellung vom Ich als Subjekt und vom Ich als Objekt erst ermöglicht, unterlaufen. Die gängigen Theorien zum Freiheitsproblem werden als bloße „Verstandestheorien“ entlarvt, die sich angesichts der Frage Freiheit oder Determinismus, Ich als Subjekt oder Ich als Objekt, dogmatisch für eine der beiden Pole entscheiden wollen und nicht wie Hegel den Gegensatz durch die geltungslogische Rekonstruktion der Problemlage selbst aufheben.

\section{VERWENDETE LITERATUR}

Peter Bieri, 1997, Analytische Philosophie des Geistes. Weinheim: Beltz. G.W.F. Hegel, 1988, Phänomenologie des Geistes [PhG]. Hamburg: Meiner. , 1991, Enzyklopädie der Wissenschaften (1830) [Enz]. Hamburg: Meiner.

Christoph Hubig, 1985, Handlung, Identität, Verstehen. Weinheim/Basel: Beltz.

Immanuel Kant, 1998, Kritik der reinen Vernunft [KrV]. Hamburg: Meiner. 1998, Grundlegung zur Metaphysik der Sitten [GMS]. Stuttgart: Reclam.

John McDowell, 2003, ,The Apperceptive I and the Empirical Self. Towards a Heterodox Reading of " Lordship and Bondage"“ in Bulletin of the Hegel Society of Great Britain 47/48, 1-16.

Pirmin Stekeler-Weithofer, 2008, „Wer ist der Herr, wer ist der Knecht? Der Kampf zwischen Denken und Handeln als Grundform jedes Selbstbewußtseins“" in Klaus Vieweg und Wolfgang Welsch (Hrsg.) He gels Phänomenologie des Geistes. Ein kooperativer Kommentar zu einem

Schlüsselwerk der Moderne. Frankfurt a.M.: Suhrkamp, 205-237. 